\title{
New actions for minimally doubled fermions and their counterterms
}

\author{
Stefano Capitani* \\ Institut für Kernphysik and HIM (Helmholtz-Institut Mainz), \\ University of Mainz, Johann-Joachim-Becher-Weg 45, D-55099 Mainz, Germany \\ E-mail: capitan@kph.uni-mainz.de
}

\begin{abstract}
Minimally doubled fermions provide a cheap and convenient way of simulating quarks which preserve chiral symmetry. It has been established that two actions of this kind (known as BoriçiCreutz and Karsten-Wilczek) require the tuning of three counterterms in order to be properly renormalized. Here we construct some more general minimally doubled actions and investigate the properties of their counterterms.
\end{abstract}

31 st International Symposium on Lattice Field Theory LATTICE 2013

July 29 - August 3, 2013

Mainz, Germany

${ }^{*}$ Speaker. 


\section{Motivation}

Minimally doubled actions provide convenient fermion lattice formulations which preserve chiral symmetry for any finite lattice spacing $a$. Two fermion flavors are the minimal amount allowed by the Nielsen-Ninomiya theorem in order to maintain, together with an exact continuous chiral symmetry (of the standard type, i.e. not Ginsparg-Wilson), other convenient properties like locality and unitarity. Such chiral fermionic formulations can still be kept ultralocal, like Wilson fermions, but at variance with the latter no tuning of masses is required, since the continuous chiral symmetry protects masses from additive renormalization.

Compared with staggered fermions, which have the same kind of $U(1)$ chiral symmetry, minimally doubled fermions are computationally slightly more expensive, however having 2 flavors instead of 4 they do not require uncontrolled extrapolations to 2 physical light flavors, and so they are ideal for $N_{f}=2$ simulations. One also avoids the complicated intertwining of spin and flavor of staggered fermions. As they are much cheaper than Ginsparg-Wilson fermions, minimally doubled fermions can be very convenient for vector-like theories like QCD. Moreover, they might be very practical for simulations of lattice QCD at finite temperature, where staggered fermions are extensively used (as a glance at the corresponding contributions in these proceedings can attest).

Two particular realizations of minimally doubled fermions have been studied in deeper detail in the last few years, the Karsten-Wilczek [1, 2] and Boriçi-Creutz [3, 4, 5, 6] actions. Since they contain only nearest-neighbor interactions, these actions are quite cheap and easy to simulate. Moreover, they allow the construction of conserved axial currents which have a simple expression (again only involving nearest-neighbor sites).

It turns out that for the massless Karsten-Wilczek (KW) and Boriçi-Creutz (BC) formulations three counterterms need to be added to the bare action in order to remove the hypercubic-breaking contributions $[7,8,9,10]$. A consistent renormalized theory can be achieved only for special values of the coefficients of the counterterms, which then have to be appropriately tuned. Once these special values are known one can carry out Monte Carlo computations of physical observables almost as easily and inexpensively as with Wilson fermions.

We present here minimally doubled actions that have the correct continuum limit (like the KW and $\mathrm{BC}$ fermions) but require fewer counterterms when appropriate values of their 2 parameters $\alpha$ and $\lambda$ are chosen. These actions can be seen as generalizations of the KW action, where the distance $2 \alpha$ (modulo $2 \pi$ ) in momentum space between the 2 poles of the quark propagator can be varied at will, like in the actions proposed in [11, 12].

The three possible counterterms for all actions presented here (including the next-to-nearestneighbor actions of Sect. 4) are the same of the standard KW action. This happens because both poles of the quark propagator still lie entirely on the temporal axis, and thus the temporal direction is always selected as the special one (irrespective of the values of $\alpha$ and $\lambda$ ), and moreover the spinorial structure of all these actions is also the same. Thus, $P$ is a symmetry, and also $C T$ [13], but $T$ and $C$ separately are violated (unless the actions are properly renormalized).

In massless quenched QCD only 2 of these counterterms are needed, the fermionic counterterm of dimension four, of the form $i d_{4}\left(g_{0}\right) \bar{\psi} \gamma_{4} D_{4} \psi$, and the counterterm of dimension three, of the form $i d_{3}\left(g_{0}\right) / a \bar{\psi} \gamma_{4} \psi$. In full QCD the gluonic part of the action can generate through internal quark loops a gluonic counterterm, of the form $d_{\mathrm{P}}\left(g_{0}\right) \sum_{\rho, \lambda} \operatorname{Tr} F_{\rho \lambda}(x) F_{\rho \lambda}(x) \delta_{\rho 4}$. 
The values of the coefficients of the counterterms for which one is able to obtain a consistent renormalized theory are functions of $\alpha$ and $\lambda$ which could vanish in some place. We indeed find that this is the case, and that there are a few curves in the space spanned by $\alpha$ and $\lambda$ for which one of the counterterms vanishes. Thus, the renormalized actions corresponding to these particular choices of the parameters require only 2 counterterms. The ultimate goal is to find actions for which all functions happen to become zero for the same values of $\alpha$ and $\lambda$. In this case no counterterms at all will be required, and one will be able to carry out consistent simulations using just the tree level actions. They will be then much cheaper than the already convenient case of (say) KW fermions.

\section{Nearest-neighbor minimally doubled actions}

We study the class of bare fermionic actions

$$
a^{4} \sum_{x} \bar{\psi}(x)\left\{\frac{1}{2} \sum_{\mu}\left[\gamma_{\mu}\left(\nabla_{\mu}+\nabla_{\mu}^{\star}\right)-i a \gamma_{4}\left(\lambda+\delta_{\mu 4}(\cot \alpha-\lambda)\right) \nabla_{\mu}^{\star} \nabla_{\mu}\right]+m_{0}\right\} \psi(x),
$$

where $\nabla_{\mu} \psi(x)=\left(U_{\mu}(x) \psi(x+a \widehat{\mu})-\psi(x)\right) / a .{ }^{1}$ These minimally doubled actions have $\mu=4$ as a special direction (like in the standard KW action), satisfy $\gamma_{5}$-hermiticity, and the interactions are only between nearest-neighbor lattice sites. The standard KW action corresponds to $\alpha=\pi / 2$.

In momentum space the Dirac operators of these fermions read, in the free case,

$$
\frac{i}{a} \sum_{\mu=1}^{4} \gamma_{\mu} \sin a p_{\mu}+\frac{i \gamma_{4}}{a}\left[\lambda \sum_{k=1}^{3}\left(1-\cos a p_{k}\right)+\cot \alpha\left(1-\cos a p_{4}\right)\right]+m_{0}
$$

and they have two zeros, located at $a \bar{p}_{1}=(0,0,0,0)$ and $a \bar{p}_{2}=(0,0,0,-2 \alpha)$, which describe two fermions of opposite chirality. For $\alpha=0$ and $\alpha=\pi$ the actions become singular $(\cot \alpha=\infty)$, and the range of $\alpha$ can be taken as $0<\alpha<\pi$.

Varying $\lambda$ does not change the location of any of the zeros of the actions, as this parameter has only the task of decoupling the 14 other fermions from the naive fermionic action (which corresponds to the first term in Eq. (2.2)), giving them a mass of order $1 / a$. However, to avoid the appearance of other doublers it must also be (at tree level) $\lambda>(1-\cos \alpha) /(2 \sin \alpha)$.

All the actions presented and considered in this article have, irrespective of the values of $\alpha$ and $\lambda$, the correct continuum limit. Since they are Wilson-like with hopping terms of only one unit of the lattice spacing $a$, they are rather cheap to simulate. The computational effort will be about a few times the one required for Wilson fermions [15].

The possibility of constructing a conserved axial current, which also has a simple form and is cheap to use in Monte Carlo simulations, constitutes one of the major advantages of using these formulations of minimally doubled fermions. Its expression for the above actions is

$$
\begin{aligned}
A_{\mu}^{\text {cons }}(x ; \alpha, \lambda)= & \frac{1}{2}\left(\bar{\psi}(x)\left(\gamma_{\mu}-i \gamma_{4}\left(\lambda+\delta_{\mu 4}(\cot \alpha-\lambda)\right)\right) \gamma_{5} U_{\mu}(x) \psi(x+a \widehat{\mu})\right. \\
& \left.+\bar{\psi}(x+a \widehat{\mu})\left(\gamma_{\mu}+i \gamma_{4}\left(\lambda+\delta_{\mu 4}(\cot \alpha-\lambda)\right)\right) \gamma_{5} U_{\mu}^{\dagger}(x) \psi(x)\right) \\
& +\frac{d_{4}\left(g_{0}\right)}{2}\left(\bar{\psi}(x) \gamma_{4} \gamma_{5} U_{4}(x) \psi(x+a \widehat{4})+\bar{\psi}(x+a \widehat{4}) \gamma_{4} \gamma_{5} U_{4}^{\dagger}(x) \psi(x)\right) .
\end{aligned}
$$

\footnotetext{
${ }^{1}$ For the expanded expressions of these actions, and of those of the next-to-nearest-neighbor actions introduced in Sect. 4, see also Ref. [14].
} 
This is an exactly conserved quantity for any finite value of the lattice spacing $a$, and only involves nearest-neighbor sites. This is particularly important, as not many fermionic formulations exist for which a conserved axial current exists and is of such a simple form.

\section{Curves of counterterm removal}

Counterterms need to be introduced to compensate (when properly tuned) for hypercubicbreaking factors. Their coefficients can be determined by making these factors disappear in 1-loop amplitudes, so that these actions are properly renormalized. The main objective of this work is to see if and when the counterterms can vanish. This means that the corresponding functional forms are missing in the 1-loop radiative corrections when special values of $\alpha$ and $\lambda$ are employed. We use Wilson's plaquette action in a general covariant gauge (where $\partial_{\mu} A_{\mu}=0$ ), and $m_{0}=0$.

Due to the non-trivial form of the denominator of the quark propagator, it is not possible to provide results with an analytic dependence on $\alpha$ or $\lambda$. The search for the special values of these parameters which remove the hypercubic-breaking factors in the 1-loop quark self-energy and vacuum polarization must then be carried out numerically. The tadpole of the self-energy however can be calculated analytically, and in a general covariant gauge is given by ${ }^{2}$

$$
T=g_{0}^{2} C_{F} \frac{Z_{0}}{2}\left(1-\frac{1}{4}(1-\xi)\right)\left(i \not p-\frac{i \gamma_{4}}{a}(3 \lambda+\cot \alpha)\right) .
$$

To carry out the calculations of the other diagrams required for the tuning of the counterterms we have used a set of computer codes written in the algebraic manipulation language FORM [19, 20], extended to include the special features of the actions presented here.

Our main results are summarized in Fig. 1, which shows the curves for which the various counterterms have a vanishing coefficient. Each counterterm can be removed, but there are no intersections between the curves of zeros, and so at least 2 counterterms are always required. ${ }^{3}$

\section{Next-to-nearest-neighbor minimally doubled actions}

It would be nice to find minimally doubled actions for which intersections between the curves of zeros exist, so that 2 (or even all) of the possible counterterms can then be removed. We introduce then interactions also between next-to-nearest-neighbor lattice sites. It could be that actions which contain interactions also at distance $2 a$ or larger have somewhat different properties, and the hope is that at the end some of these actions will not require any counterterms to be properly renormalized.

We find then useful to propose here a first example of a class of minimally doubled actions with next-to-nearest-neighbor interactions, which depend on 4 parameters: ${ }^{4}$

$$
a^{4} \sum_{x} \bar{\psi}(x)\left\{\sum_{\mu}\left[\frac{1}{2} \gamma_{\mu}\left(\nabla_{\mu}+\nabla_{\mu}^{\star}\right)-i a \gamma_{4}\left\{\frac{1}{2} f_{\mu}^{(1)} \nabla_{\mu}^{\star} \nabla_{\mu}-f_{\mu}^{(2)} \widetilde{\nabla}_{\mu}^{\star} \widetilde{\nabla}_{\mu}\right\}\right]+m_{0}\right\} \psi(x),
$$

\footnotetext{
${ }^{2}$ The quantity $Z_{0}=0.1549333 \ldots$ is an often-recurring lattice integral $[16,17,18]$.

${ }^{3}$ It is not necessary at this stage to determine the zeros with high precision, as we mainly want to show that curves of zeros exist, and see what shape they have. When a nonperturbatively renormalized action which needs just one (or no) counterterm will be found, a determination with higher precision of the relevant parameters will be then desirable.

${ }^{4}$ Here $\widetilde{\nabla}_{\mu} \psi(x)=\left(U_{\mu}(x) U_{\mu}(x+a \widehat{\mu}) \psi(x+2 a \widehat{\mu})-\psi(x)\right) /(2 a)$ is another discretization of the lattice covariant derivative, extending this time over two lattice sites.
} 


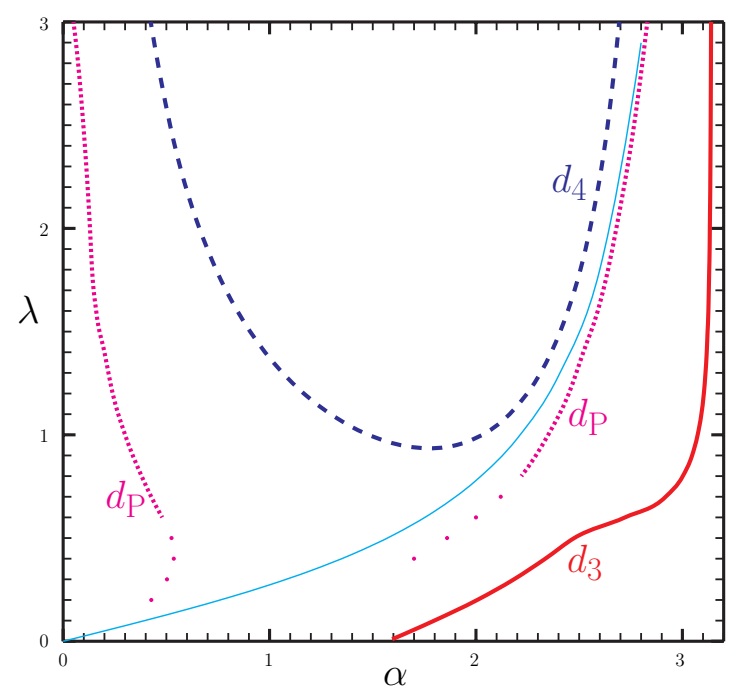

Figure 1: The curves on which the coefficients of the various counterterms vanish. Shown is also the function $\lambda=(1-\cos \alpha) /(2 \sin \alpha)$, below which additional doublers can appear (at tree-level).

where

$$
\begin{aligned}
f_{\mu}^{(1)}\left(\alpha, \lambda, \lambda^{\prime}, \rho\right) & =\lambda+2 \lambda^{\prime}+\delta_{\mu 4}\left(\left(\rho+\frac{1-\rho}{\sin ^{2} \alpha}\right) \cot \alpha-\lambda-2 \lambda^{\prime}\right) \\
f_{\mu}^{(2)}\left(\alpha, \lambda^{\prime}, \rho\right) & =\lambda^{\prime}+\delta_{\mu 4}\left(\frac{1-\rho}{2 \sin ^{2} \alpha} \cot \alpha-\lambda^{\prime}\right) .
\end{aligned}
$$

These actions satisfy $\gamma_{5}$-hermiticity, and like for the standard KW action the temporal direction is again the special one which is selected and which then breaks hypercubic symmetry. The symmetries of these actions and their possible counterterms are the same of the standard KW action.

The corresponding momentum-space actions are given in the free case by ${ }^{5}$

$$
\begin{aligned}
\frac{i}{a} \sum_{\mu=1}^{4} \gamma_{\mu} \sin a p_{\mu}+\frac{i \gamma_{4}}{a}\left\{\sum_{k=1}^{3}(\right. & \left.\lambda\left(1-\cos a p_{k}\right)+\lambda^{\prime}\left(1-\cos a p_{k}\right)^{2}\right) \\
& \left.+\cot \alpha\left(\rho\left(1-\cos a p_{4}\right)+\frac{1-\rho}{2 \sin ^{2} \alpha}\left(1-\cos a p_{4}\right)^{2}\right)\right\}+m_{0} .
\end{aligned}
$$

The parameter $\alpha$ regulates the distance between the two zeros, which are at the same positions $a \bar{p}_{1}=(0,0,0,0)$ and $a \bar{p}_{2}=(0,0,0,-2 \alpha)$ as in the nearest-neighbor actions. That there are only two zeros is certainly the case if $-3 \leq \rho \leq 1$ (as we have numerically verified), and $0<\alpha<\pi$ as before. For choices of $\rho$ outside of this range, additional zeros can in general appear, and one can still get minimally doubled actions but only for a restricted domain of $\alpha$ (whose extension depends on the value of $\rho$ ). ${ }^{6}$ Moreover, one must also respect the (tree level) condition $\lambda+2 \lambda^{\prime}>$ $-\min \left\{\sin x+\cot \alpha\left(\rho(1-\cos x)+(1-\rho)(1-\cos x)^{2} /\left(2 \sin ^{2} \alpha\right)\right)\right\} / 2$ to ensure that there are no more than two fermions.

\footnotetext{
${ }^{5}$ Notice that for $\lambda^{\prime}=0$ and $\rho=1$ one falls back to the case of the nearest-neighbor actions (2.1).

${ }^{6}$ For example, with the choice $(\alpha, \rho)=(0.1,1.1)$ and for $\vec{p}=(0,0,0)$, the action is proportional to $\gamma_{4}$, and its coefficient a function of $p_{4}$ only, which has four intersections with the $p_{4}=0$ axis.
} 
The hope with these next-to-nearest-neighbor actions is that for special choices of the parameters one could hit on renormalized actions which do not require any counterterms. The fact that there are 4 parameters, and not just 2 as in the nearest-neighbor actions, should result in many more curves on which the counterterms become zero and, above all, more chances for intersections among these curves. It could then happen that there exist some values of the parameters for which one ends up with just one counterterm, or none at all. Adequately exploring this larger parameter space is much more expensive than for the nearest-neighbor actions, and this is left for future work.

\section{Outlook}

We have shown that there are curves in the space spanned by the two parameters $\alpha$ and $\lambda$, which define the nearest-neighbor actions that we have introduced, on which some of the counterterms vanish, and on these curves the counterterms needed are fewer than the 3 required for the standard massless $\mathrm{KW}$ and $\mathrm{BC}$ actions. There are numerous choices of parameters which give just 2 counterterms, and hence only 1 in the unquenched case. Although the conclusions presented in this work arise from perturbative calculations, it is likely that also in numerical simulations the removal of some of the counterterms can be accomplished for appropriate choices of the parameters. The first task will then be to check whether the qualitative pattern of the curves that we have found here is also reproduced nonperturbatively.

In principle some intersection points could appear at the nonperturbative level. If some of the curves of zeros had indeed an intersection point, the corresponding values of $\alpha$ and $\lambda$ would provide a renormalized minimally doubled action which requires only one counterterm. Therefore, even though here using perturbation theory this has not occurred, it could well be the case that nonperturbatively an intersection point does exist. This would make possible to simulate renormalized minimally doubled actions with at most one counterterm.

It is also possible that even cleverer minimally doubled actions can be constructed, which would be able to remove all possible counterterms. In this case Monte Carlo simulations employing just the bare action will be sufficient for the extraction of significant physical results, and no tuning of counterterms will be required to simulate such an action.

But even when it is not possible to remove all counterterms, it is always useful to be able to accomplish a reduction in the dimensionality of the parameter space of their coefficients, as this makes their numerical evaluation easier. In particular, if there is only one counterterm left, it is much simpler to carry out the determination of its coefficient, because one has to deal with just a one-dimensional space instead of a multi-parameter one.

In any case, apart from the removal of counterterms, it is always useful to have as many different minimally doubled actions as possible and keep on trying to construct new ones, because some of them could turn out to possess better theoretical or practical properties. The actual amount of important quantities such as the mass difference between the $\pi^{ \pm}$and the $\pi^{0}$, or of mass splittings within otherwise degenerate multiplets, could turn out to be rather small for a few of these actions and not for all other ones. In general it can be convenient to have minimally doubled actions where the distance between the two poles of the quark propagator can be arbitrarily varied. Special values of this distance could also provide actions which could turn out to be more advantageous for Monte Carlo simulations (in that for instance they minimize some artefacts peculiar to these actions). 
Thus, this work can also be considered as an inspiration to undertake further searches for new minimally doubled actions which require a reduced number of counterterms, and possibly (in the best of cases) none at all. Further theoretical investigations, and the twisted-ordering method presented in [21, 22, 23] (see also the overview in [24]), which can be useful for constructing other minimally doubled actions, could suggest how to steer these searches also in new directions.

The next-to-nearest-neighbor actions that we have introduced could also be taken as a starting point for a special direction in this undertaking, especially if it turns out that next-to-nearestneighbor interactions possses some fundamental feature different from the nearest-neighbor case, in particular with respect to the type of counterterms which can arise.

\section{References}

[1] L. H. Karsten, Phys. Lett. B104 (1981) 315.

[2] F. Wilczek, Phys. Rev. Lett. 59 (1987) 2397.

[3] M. Creutz, JHEP 0804 (2008) 017 [arXiv:0712.1201 [hep-lat]].

[4] A. Boriçi, Phys. Rev. D 78 (2008) 074504 [arXiv:0712.4401 [hep-lat]].

[5] M. Creutz, PoS LATTICE 2008 (2008) 080 [arXiv:0808.0014 [hep-lat]].

[6] A. Boriçi, PoS LATTICE 2008 (2008) 231 [arXiv:0812.0092 [hep-lat]].

[7] S. Capitani, J. Weber and H. Wittig, Phys. Lett. B 681 (2009) 105 [arXiv:0907.2825 [hep-lat]].

[8] S. Capitani, J. Weber and H. Wittig, PoS LATTICE 2009 (2009) 075 [arXiv:0910.2597 [hep-lat]].

[9] S. Capitani, M. Creutz, J. Weber and H. Wittig, JHEP 1009 (2010) 027 [arXiv:1006.2009 [hep-lat]].

[10] S. Capitani, M. Creutz, J. Weber and H. Wittig, PoS LATTICE 2010 (2010) 093 [arXiv:1010.0110 [hep-lat]].

[11] M. Creutz, PoS LATTICE 2010 (2010) 078 [arXiv:1009.3154 [hep-lat]].

[12] M. Creutz, Acta Phys. Slov. 61 (2011) 1 [arXiv:1103.3304 [hep-lat]].

[13] P. F. Bedaque, M. I. Buchoff, B. C. Tiburzi and A. Walker-Loud, Phys. Lett. B 662 (2008) 449 [arXiv:0801.3361 [hep-lat]].

[14] S. Capitani, arXiv:1307.7497 [hep-lat].

[15] S. Capitani, J. Weber and H. Wittig, these proceedings.

[16] A. González-Arroyo and C. P. Korthals-Altes, Nucl. Phys. B 205 (1982) 46.

[17] R. K. Ellis and G. Martinelli, Nucl. Phys. B 235 (1984) 93 [Erratum-ibid. B 249 (1985) 750].

[18] S. Capitani, Phys. Rept. 382 (2003) 113 [arXiv:hep-lat/0211036].

[19] J. A. M. Vermaseren, arXiv:math-ph/0010025.

[20] J. A. M. Vermaseren, Nucl. Phys. Proc. Suppl. 183 (2008) 19 [arXiv:0806.4080 [hep-ph]].

[21] M. Creutz and T. Misumi, Phys. Rev. D 82 (2010) 074502 [arXiv:1007.3328 [hep-lat]].

[22] T. Misumi, M. Creutz and T. Kimura, PoS LATTICE 2010 (2010) 260 [arXiv:1010.3713 [hep-lat]].

[23] T. Kimura, S. Komatsu, T. Misumi, T. Noumi, S. Torii and S. Aoki, JHEP 1201 (2012) 048 [arXiv:1111.0402 [hep-lat]].

[24] T. Misumi, PoS LATTICE 2012 (2012) 005 [arXiv:1211.6999 [hep-lat]]. 